Mathematical Sciences and Applications

E-NOTES

MATHEMATICAL

SCIENCES

AND APPLICATIONS

E-NOTES

https://doi.org/10.36753/msaen.1025072

10 (3) 135-145 (2022) - Research Article

ISSN: 2147-6268

(C)MSAEN

\title{
A Trigonometric Approach to Time Fractional FitzHugh-Nagumo Model on Nerve Pulse Propagation
}

\author{
Berat Karaağac
}

\begin{abstract}
The aim of this paper is to put on display the numerical solutions and dynamics of time fractional Fitzhugh-Nagumo model, which is an important nonlinear reaction-diffusion equation. For this purpose, finite element method based on trigonometric cubic B-splines are used to obtain numerical solutions of the model. In this model, the derivative which is fractional order is taken in terms of Caputo. Thus, time dicretization is made using $L 1$ algorithm for Caputo derivative and space discretization is made using trigonometric cubic B- spline basis. Also, the non-linear term in the model is linearized by the Rubin Graves type linearization. The error norms are calculated for measuring the accuracy of the finite element method. The comparison of numerical and exact solutions are exhibited via tables and graphics.
\end{abstract}

Keywords: Time fractional Fitzhugh-Nagumo model; finite element method; collocation; trigonometric B-splines

AMS Subject Classification (2020): Primary: 65L60 ; Secondary: 65A05; 41A15.

\section{Introduction}

The roots of Fractional calculus (FC) dates back to 1965. This date is not far from the emerging date of traditional calculus. Nonetheless; though its fascinating nature and contribution of mathematicians, physicists and engineers, it has not been a huge attraction. Recent decades have seen a dramatically accelerating pace in the development of fractional calculus due to frequently appearing in various applications in fields of biomechanics, viscoelasticity, control theory, aerodynamics, physics and engineering and so on [1,2]. The popularity of FC has attracted many researchers from all over the world, thus, this attraction produced research papers and books covering all areas of science and motivated and development of numerical methods [3, 4]. Especially, since fractional derivatives are more convenient and economical, solving fractional order differential equations has a vital role in modeling various phenomena. One can get a brief glimpse to numerical methods for solving fractional differential equations in Refs[5-14].

In this paper, Time fractional Fitzhugh-Nagumo model is going to be considered via initial and boundary conditions. In order to obtain numerical solutions a framework of combination collocation method and finite element method (FEM) based on cubic trigonometric B-spline basis will be used. The error norms will be calculated 
to know how well accurate the exact solutions and numerical ones close to each other. Comparison of exact and numerical results and dynamics of the solutions is going to be presented via tables and graphics.

\section{Time fractional Fitzhugh-Nagumo model and Application of the method}

The FitzHugh-Nagumo model [18]

$$
\begin{aligned}
& \varepsilon \frac{\partial u(x, t)}{\partial t}=\Psi(u(x, t))-v(x, t)+I, \\
& \frac{\partial v(x, t)}{\partial t}=u(x, t)-\zeta v(x, t)
\end{aligned}
$$

where $\varepsilon$ and $\zeta$ are positive constants and $\Psi(u(x, t))=u(x, t)(1-u(x, t))(u(x, t)-r)$ for $r \in\left(0, \frac{1}{2}\right)$. The model takes its name from R. FitzHugh [15] and J. Nagumo [16] who proposed a model for emulating the current signal observed in a living organism's excitable cells in 1961. A lipid bilayer membrane distinct nerve cells from the extracellular region. When the cells do not run the signals, there are a a potential difference. This potential difference is known as resting potential of cells. Positively charged sodium and the potassium ions and negatively charged protein ions keep in existence of resting potential. When there is a external disturbation to the cells, depolarization and repolarization process begins. Depolarization rises up spikes toward a positive value, depolarization passes to resting potential. In a simple description, the FitzHugh-Nagumo model is a simplified model of activation and deactivation dynamics in a spiking neuron [17]. In literature, $I$ is a constant external stimulus. $u(x, t)$ and $v(x, t)$ are unknowns and they measure the potential difference across the cell membrane transmembrane currents which influence the tendency of the cell to regain before being able to fire again, respectively [18]. It will be useful to explain that many variations of FitzHugh-Nagumo model have been derived from the original one.

There are various approaches to FitzHugh-Nagumo model. One can read and get information in [19-22] and there in. For the reason that the main issue of this paper, it will be important to take a quick glance at fractional FitzHugh-Nagumo model; Liu et al. [23] have considered the model in two dimensional and obtained solutions via implicit numerical method. Kumar et al. [24] have focused on solutions of the model using $q$-homotopy analysis approach and Laplace transform approach. Injrou et al. [25] proposed a finite difference scheme to obtain numerical solutions of the model.

In this paper, a reduced problem of FitzHugh-Nagumo model in fractional form will be taken into consideration. As the starting point, time fractional nonlinear reaction-diffusion equations of the form is considered as following [25]

$$
D_{t}^{\gamma} u_{t}(x, t)=\kappa u_{x x}(x, t)+\Psi(u(x, t))
$$

where $x$ and $t$ denote space and time derivative, respectively. $\kappa$ is a physical constant which represents diffusion coefficient , $\Psi$ accounts for all local reactions and $\gamma$ is the fractional order and $0<\gamma<1$.In order to calculate wave solutions, the function $\Psi(u(x, t))$ has to be specified; if $\kappa=1$ and $\Psi(u(x, t))=u(x, t)(1-u(x, t))(u(x, t)-r)$, the equation (2.1) is called as time fractional Fitzhugh-Nagumo model. Thus, time fractional initial-boundary Fitzhugh-Nagumo model [24, 26, 27] will be considered and it is formulated as

$$
\begin{aligned}
& D_{t}^{\gamma} u(x, t)=\kappa u_{x x}(x, t)+\mu u(x, t)(1-u(x, t))(u(x, t)-r), \\
& u\left(x_{L}, t\right)=f_{1}(t), \quad u\left(x_{R}, t\right)=f_{2}(t), \quad t \in[0, T] \\
& u_{x}\left(x_{L}, t\right)=g_{1}(t), \quad u_{x}\left(x_{R}, t\right)=g_{2}(t), \quad x \in\left[x_{L}, x_{R}\right] \\
& u(x, 0)=h(x) .
\end{aligned}
$$

As highlighted above, there are several variants of the FitzHugh-Nagumo equations. In this subsection finite element collocation method will be considered for the model given in (2.2) with its initial and boundary conditions. In order to compute the numerical solutions of the FitzHugh-Nagumo model via FEM on fixed points on a specific interval $\left[x_{L}, x_{R}\right]$, the interval should be divided $N$ subelements with $h=\frac{x_{R}-x_{L}}{N}$ such that

$$
x_{L}=x_{0}<x_{1}<x_{2}<\cdots<x_{N-2}<x_{N-1}<x_{N}=x_{R}
$$

where $\left.\left\{x_{m}\right\}\right|_{m=0} ^{N}$ are distinct grid points and number of grid points is $(N+1)$. The procedure for obtaining a numerical scheme is to compute numerical solutions define an approximate solution for the mentioned model. Let 
$w(x, t)$ is an approximate solution to exact solution $u(x, t)$ is determined as follows

$$
u(x, t) \approx w(x, t)=\sum_{j=-1}^{N+1} \delta_{j}(t) T B_{j}^{3}(x)
$$

where $\delta_{j}(t)$ are time dependent parameters which will be determined for obtaining numerical solutions at the point $\left(x_{j}, t_{n}\right)$ and for this problem $T B_{j}^{3}(x)$ are cubic trigonometric B-spline basis given in [28] as follows

$$
T B_{m}^{3}(x)=\frac{1}{\phi} \begin{cases}p^{2}\left(x_{m-2}\right)-p^{2}\left(x_{m-2}\right) p\left(x_{m}\right), & x_{m-2}<x<x_{m-1} \\ -p\left(x_{m-2}\right) p\left(x_{m+1}\right) p\left(x_{m-1}\right)-p\left(x_{m+2}\right) p^{2}\left(x_{m-1}\right), & x_{m-1}<x<x_{m} \\ p\left(x_{m-2}\right) p^{2}\left(x_{m+1}\right)+p\left(x_{m+2}\right) p\left(x_{m-1}\right) p\left(x_{m+1}\right), & x_{m}<x<x_{m+1} \\ +p^{2}\left(x_{m+2}\right) p\left(x_{m}\right), & \\ -p^{3}\left(x_{m+2}\right) & x_{m+1}<x<x_{m+2} \\ 0 & \text { otherwise }\end{cases}
$$

where $p(x m)=\sin \left(\frac{x-x_{m}}{2}\right)$ and $\phi=\sin \left(\frac{h}{2}\right) \sin (h) \sin \left(\frac{3 h}{2}\right)$ for $m=0,1,2, \ldots, N . T B_{j}(x)$ basis are zero out of interval $\left[x_{m-2}, x_{m+2}\right]$. On the ground of local support property of basis $T B_{m}(x)$, the approximate solution can be defined over a sub interval $\left[x_{m}, x_{m+1}\right]$ as

$$
w(x, t)=\sum_{j=m-1}^{m+2} \delta_{j}(t) T B_{j}^{3}(x)
$$

Now, when the value of $w(x, t)$ at point $x_{m}$ is expressed as $w_{m}^{n}$, it and its required derivatives can be obtained with some calculations as follows

$$
\begin{aligned}
& w_{m}^{n}=\alpha_{1} \delta_{m-1}(t)+\alpha_{2} \delta_{m}(t)+\alpha_{1} \delta_{m+1}(t), \\
& \left(w_{m}^{n}\right)^{\prime}=\beta_{1} \delta_{m-1}(t)+\beta_{2} \delta_{m+1}(t), \\
& \left(w_{m}^{n}\right)^{\prime \prime}=\eta_{1} \delta_{m-1}(t)+\eta_{2} \delta_{m}(t)+\eta_{1} \delta_{m+1}(t)
\end{aligned}
$$

where

$$
\begin{array}{ll}
\alpha_{1}=\sin ^{2}\left(\frac{h}{2}\right) \csc (h) \csc \left(\frac{3 h}{2}\right), & \alpha_{2}=\frac{2}{1+\cos (h)}, \\
\beta_{1}=-\frac{3}{4} \csc \left(\frac{3 h}{2}\right), & \beta_{2}=\frac{3}{4} \csc \left(\frac{3 h}{2}\right), \\
\eta_{1}=\frac{3\left((1+3 \cos (h)) \csc ^{2}\left(\frac{h}{2}\right)\right)}{16\left(2 \cos \left(\frac{h}{2}\right)+\cos \left(\frac{3 h}{2}\right)\right)}, & \eta_{2}=\frac{3 \cot ^{2}\left(\frac{h}{2}\right)}{2+4 \cos (h)} .
\end{array}
$$

The solution of the problem given in (2.2) will be obtained by substituting approximate solution and its derivatives into discretization of the problem. Thus, for a first task, Fitzhugh-Nagumo model should be discretized.

\section{Discretization of the Fitzhugh-Nagumo model and Application of the method}

In order to compute numerical solutions of Fitzhugh-Nagumo model, it is crucial to discritize the model and obtain recursive equation system i.e numerical scheme. For this purpose, the derivatives according to space will be discretize using Crank-Nicolson method, which is a average in time, by reason of obtaining the second order accurate and the fractional order derivative according to time will be discretized using $L 1$ algorithm [29] as follows

$$
D_{t}^{\gamma} f\left(t_{m}\right)=\frac{(\Delta t)^{-\gamma}}{\Gamma(2-\gamma)} \sum_{k=0}^{n-1} b_{k}^{\gamma}\left[f\left(t_{n-k}\right)-f\left(t_{n-1-k}\right)\right]
$$

where $b_{k}^{\gamma}=(k+1)^{1-\gamma}-k^{1-\gamma}, t_{n}=n \Delta t(n=0,1.2, \ldots, M)$, and final time $T=M \Delta t$

When Crank-Nicolson method and $L 1$ algorithm applied to Fitzhugh-Nagumo model given in (2.2), it yields;

$$
\begin{gathered}
\frac{(\Delta t)^{-\gamma}}{\Gamma(2-\gamma)} \sum_{k=0}^{n-1} b_{k}^{\gamma}\left[u^{n-k}-u^{n-1-k}\right]=\frac{\kappa}{2}\left(\left(u_{x x}\right)^{n+1}+\left(u_{x x}\right)^{n}\right)+\frac{\mu}{2}\left(\left(u^{2}\right)^{n+1}+\left(u^{2}\right)^{n}\right) \\
+\frac{\mu r}{2}\left(u^{n+1}+u^{n}\right)+\frac{\mu}{2}\left(\left(u^{3}\right)^{n+1}+\left(u^{3}\right)^{n}\right)-\frac{\mu r}{2}\left(\left(u^{2}\right)^{n+1}+\left(u^{2}\right)^{n}\right)
\end{gathered}
$$


From this point of view, nonlinear terms seen in $(3.1)$ at $(n+1)$ time level will be linearized via Rubin-Graves linearization method as following way;

$$
\begin{aligned}
& \left(u^{2}\right)^{n+1}=u^{n+1} u^{n}+u^{n} u^{n+1}-u^{n} u^{n} \\
& \left(u^{3}\right)^{n+1}=u^{n+1} u^{n} u^{n}+u^{n} u^{n+1} u^{n}+u^{n} u^{n} u^{n+1}-2 u^{n} u^{n} u^{n} .
\end{aligned}
$$

Putting approximate solution and its derivatives (2.3) into (3.1), and a bit of simple arrangements gives a recursive equation system as follows

$$
\begin{aligned}
\delta_{m-1}^{n+1}[ & \left.\alpha_{1}+\frac{S}{2}\left(\mu \alpha_{1} \rho_{1}-\kappa \eta_{1}\right)\right]+\delta_{m}^{n+1}\left[\alpha_{2}+\frac{S}{2}\left(\mu \alpha_{2} \rho_{1}-\kappa \eta_{2}\right)\right]+\delta_{m+1}^{n+1}\left[\alpha_{1}+\frac{S}{2}\left(\mu \alpha_{1} \rho_{1}-\kappa \eta_{1}\right)\right] \\
= & \delta_{m-1}^{n+1}\left[\alpha_{1}+\frac{S}{2}\left(\alpha_{1} \rho_{2}+\kappa \eta_{1}\right)\right]+\delta_{m}^{n+1}\left[\alpha_{2}+\frac{S}{2}\left(\alpha_{2} \rho_{2}+\kappa \eta_{2}\right)\right]+\delta_{m+1}^{n+1}\left[\alpha_{1}+\frac{S}{2}\left(\alpha_{1} \rho_{2}+\kappa \eta_{1}\right)\right] \\
& -\sum_{k=1}^{n-1} b_{k}^{\gamma}\left[\left(\alpha_{1} \delta_{m-1}^{n-k}+\alpha_{2} \delta_{m}^{n-k}+\alpha_{1} \delta_{m+1}^{n-k}\right)-\left(\alpha_{1} \delta_{m-1}^{n-1-k}+\alpha_{2} \delta_{m}^{n-1-k}+\alpha_{1} \delta_{m+1}^{n-1-k}\right)\right] .
\end{aligned}
$$

The sub indexes seen in (3.2) are $m=0,1,2, \ldots, N$ and $t=0,1,2, \ldots, M$. Here, $T$ is final time and $\Delta t$ is time step such as $T=M . \Delta t$ coefficients of unknowns seen in recursive equation system are $S=(\Delta t)^{\gamma} \Gamma(2-\gamma)$, $\rho_{1}=3\left(u^{n}\right)^{2}-2 r u^{n}-2 u^{n}+r$ and $\rho_{2}=\mu u^{n}\left(u^{n}-r\right)$.

\subsection{Boundary condition at $x=x_{L}$}

As in with the finite difference method, boundary conditions at boundary of the interval are so essential in applying Finite element method, the numerical scheme obtained via FEM have to satisfy predefined conditions applied on their boundary.

When one glimpse at the system in (3.2), it can be realized that systems consist of $(N+1)$ equations with $(N+3)$ unknowns. For a solvable system, there are two ways, first one is adding two equation into the system and the second one is eliminating two unknown from the system. For two choice, boundary conditions will be used to achieve to aim. In this section, eliminating two unknown choice will apply. The boundary condition $u\left(x_{L}, t\right)=f_{1}(t)$ can be written using approximate solution at grid $\left(x_{m}, t_{n}\right)$ as

$$
u\left(x_{m}, t_{n}\right)=\alpha_{1} \delta_{m-1}(t)+\alpha_{2} \delta_{m}(t)+\alpha_{1} \delta_{m+1}(t), \quad m=0,1,2, \ldots, N
$$

Equation (3.3) is rewritten by $m=0$ for right boundary

$$
f_{1}(t)=\alpha_{1} \delta_{-1}(t)+\alpha_{2} \delta_{0}(t)+\alpha_{1} \delta_{1}(t) .
$$

Thus this gives;

$$
\delta_{-1}(t)=\frac{f_{1}(t)}{\alpha_{1}}-\frac{\alpha_{2}}{\alpha_{1}} \delta_{0}(t)-\delta_{1}(t) .
$$

\subsection{Boundary condition at $x=x_{R}$}

When the boundary condition $u\left(x_{R}, t\right)=f_{2}(t)$ is put into equation (3.3) with $m=N$, it yields;

$$
f_{2}(t)=\alpha_{1} \delta_{N-1}(t)+\alpha_{2} \delta_{N}(t)+\alpha_{1} \delta_{N+1}(t) .
$$

Thus,

$$
\delta_{N+1}(t)=\frac{f_{2}(t)}{\alpha_{1}}-\frac{\alpha_{2}}{\alpha_{1}} \delta_{N}(t)-\delta_{N-1}(t) .
$$

When substituting (3.4) and (3.5) into recursive system (3.2), there will be a system consisting of $(N+1)$ equations with $(N+1)$ unknowns. When recursive system $(3.2)$ is used with boundary conditions, it gives following 
numerical scheme which will derive numerical solutions as follows

$$
\begin{aligned}
& \left\{\begin{array}{l}
\delta_{0}^{n+1}(t)\left[\kappa \frac{S}{2 \alpha_{1}}\left(\alpha_{2} \eta_{1}-\alpha_{1} \eta_{2}\right)\right]=\delta_{0}^{n}(t)\left[-\kappa \frac{S}{2 \alpha_{1}}\left(\alpha_{2} \eta_{1}-\alpha_{1} \eta_{2}\right)\right] \\
-\sum_{k=1}^{n-1} b_{k}^{\gamma}\left[u_{0}^{n-k}-u_{0}^{n-1-k}\right]+\Upsilon_{1},
\end{array}\right. \\
& \left\{\begin{array}{l}
\delta_{m-1}^{n+1}\left[\alpha_{1}+\frac{S}{2}\left(\mu \alpha_{1} \rho_{1}-\kappa \eta_{1}\right)\right]+\delta_{m}^{n+1}\left[\alpha_{2}+\frac{S}{2}\left(\mu \alpha_{2} \rho_{1}-\kappa \eta_{2}\right)\right] \\
+\delta_{m+1}^{n+1}\left[\alpha_{1}+\frac{S}{2}\left(\mu \alpha_{1} \rho_{1}-\kappa \eta_{1}\right)\right] \\
=\delta_{m-1}^{n}\left[\alpha_{1}+\frac{S}{2}\left(\alpha_{1} \rho_{2}+\kappa \eta_{1}\right)\right]+\delta_{m}^{n}\left[\alpha_{2}+\frac{S}{2}\left(\alpha_{2} \rho_{2}+\kappa \eta_{2}\right)\right]+ \\
\delta_{m+1}^{n}\left[\alpha_{1}+\frac{S}{2}\left(\alpha_{1} \rho_{2}+\kappa \eta_{1}\right)\right] \\
-\sum_{k=1}^{n-1} b_{k}^{\gamma}\left[\left(\alpha_{1} \delta_{m-1}^{n-k}+\alpha_{2} \delta_{m}^{n-k}+\alpha_{1} \delta_{m+1}^{n-k}\right)-\left(\alpha_{1} \delta_{m-1}^{n-1-k}+\alpha_{2} \delta_{m}^{n-1-k}+\alpha_{1} \delta_{m+1}^{n-1-k}\right)\right]
\end{array}\right. \\
& \left\{\begin{array}{l}
\delta_{N}^{n+1}(t)\left[\kappa \frac{S}{2 \alpha_{1}}\left(\alpha_{2} \eta_{1}-\alpha_{1} \eta_{2}\right)\right]=\delta_{0}^{n}(t)\left[-\kappa \frac{S}{2 \alpha_{1}}\left(\alpha_{2} \eta_{1}-\alpha_{1} \eta_{2}\right)\right] \\
-\sum_{k=1}^{n-1} b_{k}^{\gamma}\left[u_{N}^{n-k}-u_{N}^{n-1-k}\right]+\Upsilon_{2} .
\end{array}\right. \\
& m=0 \\
& 1<m<N-1 \\
& m=N
\end{aligned}
$$

Now, the system (3.6) is solvable. Of course, (3.6) can be given in the form of a matrix in briefly such as

$$
A \Lambda^{n+1}=B \Lambda^{n}+\Upsilon
$$

where vector $\Lambda=\left\{\delta_{0}, \delta_{1}, \ldots, \delta_{N-1}, \delta_{N}\right\}$ and vector $\Upsilon$ consist of boundary terms and values which produced via sum term. Using (3.7), the unknown vector $\Lambda^{n+1}$ will be determined by values of vector $\Lambda^{n}$.

\section{Initial State}

All recursive formulae must always state on initial terms. In this section, an initial vector must be derived. Thus, subsequent terms of the sequence can be found via initial vector. The initial vector $\Lambda^{(0)}=\left\{\delta_{0}^{(0)}, \delta_{1}^{(0)}, \ldots, \delta_{N-1}^{(0)}, \delta_{N}^{(0)}\right\}$ will be computed using the initial condition $u(x, 0)=h(x)$. For this process, approximate solution and its derivatives are used at point $x_{m}$ as follows;

$$
u\left(x_{m}, 0\right)=w\left(x_{m}, 0\right)=h\left(x_{m}\right), \quad m=0,1,2, \ldots, N
$$

When (4.1) is written in an explicit way, one can see that the system is composed of $(N+1)$ equations with $(N+3)$ unknowns. In order to eliminate two unknowns $\delta_{-1}^{(0)}$ and $\delta_{N+1}^{(0)}$ from the system, first derivative according to space variable will be used such that

$$
\begin{array}{lr}
\left(w_{m}^{(0)}\right)^{\prime}=\beta_{1} \delta_{-1}^{(0)}+\beta_{2} \delta_{1}^{(0)}=h\left(x_{0}\right), & \text { for } i=0 \\
\left(w_{m}^{(0)}\right)^{\prime}=\beta_{1} \delta_{N-1}^{(0)}+\beta_{2} \delta_{N+1}^{(0)}=h\left(x_{N}\right), & \text { for } i=N
\end{array}
$$

Thus,(4.2) yields

$$
\begin{aligned}
& \delta_{-1}^{(0)}=\frac{1}{\beta_{1}}\left(h\left(x_{0}\right)-\beta_{2} \delta_{1}^{(0)}\right), \\
& \delta_{N+1}^{(0)}=\frac{1}{\beta_{2}}\left(h\left(x_{N}\right)-\beta_{1} \delta_{N-1}^{(0)}\right) .
\end{aligned}
$$

At the end of process, all calculations result in a system involving $(N+1)$ equations with $(N+1)$ unknowns. Solving the system results in deriving initial vector $\Lambda^{(0)}$. 


\section{Numerical Approximation}

In this section, the cubic trigonometric B-splines will be used to calculate the numerical solutions of FitzHughNagumo model. Hence, accuracy and efficiency of the method will be tested of proposed numerical scheme. So as to calculate error norms, following formulas will be used;

$$
L_{2}=\sqrt{h \sum_{m=0}^{N}\left|u_{m}-w_{m}\right|^{2}}, \quad L_{\infty}=\max _{m}\left|u_{m}-w_{m}\right| .
$$

\subsection{Case 1:}

In this section, we apply the proposed technique on two cases. Consider the following time fractional FitzHughNagumo model given in (2.2) under the following conditions $\kappa=\mu=1, r=4 / 10$, Thus, the problem is translated into following form;

$$
\begin{aligned}
& D_{t}^{\gamma} u(x, t)=u_{x x}(x, t)+u(x, t)(1-u(x, t))(u(x, t)-4 / 10), \\
& \left\{\begin{array}{l}
u\left(x_{L}, t\right)=f_{1}(t), \quad u\left(x_{R}, t\right)=f_{2}(t), \\
u_{x}\left(x_{L}, t\right)=g_{1}(t), \quad u_{x}\left(x_{R}, t\right)=g_{2}(t), \quad(x, t)=[0,1] \times[0,1] \\
u(x, 0)=h(x)
\end{array}\right.
\end{aligned}
$$

where $h(x)=1 / e^{-\frac{x}{\sqrt{2}}}$ and exact solution of the model is

$$
\begin{aligned}
& u(x, t)=\frac{1}{e^{-\frac{x}{\sqrt{2}}}}-\frac{t^{\gamma}(2 r-1) e^{\frac{x}{\sqrt{2}}}}{2 \Gamma(\gamma+1)\left(1+e^{\frac{x}{\sqrt{2}}}\right)^{2}}-\frac{t^{2 \gamma}(2 r-1)^{2}\left(e^{\frac{x}{\sqrt{2}}}-1\right)}{4 \Gamma(2 \gamma+1)\left(1+e^{\frac{x}{\sqrt{2}}}\right)^{3}} \\
& -\frac{t^{3 \gamma}(2 r-1)^{3} e^{\frac{x}{\sqrt{2}}}\left(e^{\sqrt{2} x}+4 e^{\frac{x}{\sqrt{2}}}+1\right)}{16 \Gamma(2 \gamma+1)\left(1+e^{\frac{x}{\sqrt{2}}}\right)^{4}}+\frac{t^{4 \gamma}(2 r-1)^{4} e^{\frac{x}{\sqrt{2}}}\left(-11 e^{\sqrt{2} x}+e^{\frac{3 x}{\sqrt{2}}}+11 e^{\frac{x}{\sqrt{2}}}-1\right)}{96 \Gamma(2 \gamma+1)\left(1+e^{\frac{x}{\sqrt{2}}}\right)^{5}} .
\end{aligned}
$$

Boundary conditions can be seen taking $x=x_{L}$ and $x=x_{R}$ at exact solution.

As the first example, the interval which problem discussed on is chosen as $\Omega=[0,1] \times[0,1]$. The numerical solutions of (5.1) for different values of space step partition $N$ and time step $\Delta t$ and presented in Table 5.1-2 for $\gamma=0.25$. Then different values for fractional order derivative are presented in Table 3 for $N=800$ and $\Delta t=0.001$. In last table i.e Table 4, comparisons are presented between [25] and present method for $\gamma=0.9$ and different values of time step $\Delta t$.

It can be seen clearly from the table 5.1-2 that, the error norms $L_{2}$ and $L_{\infty}$ are getting smaller with increasing number of space and time discretization. So that, for $N=50$ and $\Delta t=1 / 10, L_{2}=2.06214883 \times 10^{-5}, L_{\infty}=$ $3.53959227 \times 10^{-5}$ and for $N=800$ and $\Delta t=1 / 1000, L_{2}=1.26949713 \times 10^{-5}, L_{\infty}=1.70531113 \times 10^{-5}$. We can conclude that the finite element method using trigonometric cubic splines derives quite accurate solutions to exact ones. Additionally, the results given in Table 4 are calculated using "mean absolute error" given in [25]. It can be seen from the table 4 , the error norms which are obtained using trigonometric cubic splines are in agreement with the error norms given in [25] and obtained more accurate solutions for many values of $\Delta t$.

Table 1. Case 1: Error norms $L_{2}$ and $L_{\infty}$ of Time fractional FitzHugh-Nagumo model for different values of $\Delta t$ and $N$.

\begin{tabular}{cclll}
\hline \hline$\gamma=0.25$ & \multicolumn{2}{c}{$\Delta t=0.01$} & \multicolumn{2}{c}{$\Delta t=0.01$} \\
\hline$N$ & $L_{2} \times 10^{5}$ & $L_{\infty} \times 10^{5}$ & $L_{2} \times 10^{5}$ & $L_{\infty} \times 10^{5}$ \\
\hline 50 & 2.06214883 & 3.53959227 & 1.34963953 & 1.89004865 \\
100 & 2.01958305 & 3.44947107 & 1.28629959 & 1.80653553 \\
200 & 2.00910969 & 3.42689595 & 1.27040051 & 1.78548711 \\
400 & 2.00650198 & 3.42151241 & 1.26641599 & 1.78020364 \\
800 & 2.00585031 & 3.42009868 & 1.26541792 & 1.77888708 \\
\hline \hline
\end{tabular}


Table 2. Case 1: Error norms $L_{2}$ and $L_{\infty}$ of Time fractional FitzHugh-Nagumo model for different values of $\Delta t$ and $N$.

\begin{tabular}{lllll}
\hline \hline$\gamma=0.25$ & \multicolumn{2}{c}{$\Delta t=0.05$} & \multicolumn{2}{c}{$\Delta t=0.001$} \\
\hline$N$ & $L_{2} \times 10^{5}$ & $L_{\infty} \times 10^{5}$ & $L_{2} \times 10^{-5}$ & $L_{\infty} \times 10^{5}$ \\
\hline 50 & 1.31240768 & 1.84955706 & 1.28811292 & 1.81625015 \\
100 & 1.24911235 & 1.76581920 & 1.22486871 & 1.73269800 \\
200 & 1.23322360 & 1.74499717 & 1.20899298 & 1.71185235 \\
400 & 1.22924146 & 1.73972543 & 1.20501403 & 1.70662887 \\
800 & 1.22824392 & 1.73841335 & 1.20401729 & 1.70531113 \\
\hline \hline
\end{tabular}

Table 3. Case 1: Error norms $L_{2}$ and $L_{\infty}$ of Time fractional FitzHugh-Nagumo model for different values of $\Delta t$ and $N=800$.

\begin{tabular}{lllll}
\hline \hline$N=800$ & \multicolumn{2}{c}{$\Delta t=0.01$} & \multicolumn{2}{c}{$\Delta t=0.001$} \\
\hline$\gamma$ & $L_{2} \times 10^{5}$ & $L_{\infty} \times 10^{5}$ & $L_{2} \times 10^{5}$ & $L_{\infty} \times 10^{5}$ \\
0.1 & 1.10005315 & 1.58375582 & 1.04760205 & 1.49860510 \\
0.3 & 1.30542358 & 1.83538958 & 1.24669134 & 1.75903888 \\
0.5 & 1.41379340 & 1.96531926 & 1.36063388 & 1.89242299 \\
0.8 & 1.40460709 & 1.92744306 & 1.33323670 & 1.82888194 \\
0.9 & 1.33077714 & 1.82130939 & 1.26949713 & 1.73682731 \\
\hline \hline
\end{tabular}

Table 4. Case 1: A comparison between error norms $L_{2}$ and $L_{\infty}$ for Time fractional FitzHugh-Nagumo model

\begin{tabular}{lll}
\hline \hline$\gamma=0.9$ & Present method & {$[25]$} \\
\hline$\Delta t$ & $M A E$ & $M A E$ \\
$1 / 10$ & $1.86895787 \times 10^{-5}$ & $5.35 \times 10^{-5}$ \\
$1 / 20$ & $1.51876756 \times 10^{-5}$ & $2.81 \times 10^{-5}$ \\
$1 / 40$ & $1.33900185 \times 10^{-5}$ & $1.48 \times 10^{-5}$ \\
$1 / 80$ & $1.25240772 \times 10^{-5}$ & $7.74 \times 10^{-6}$ \\
$1 / 120$ & $1.22481462 \times 10^{-5}$ & $4.04 \times 10^{-6}$ \\
\hline \hline
\end{tabular}

\subsection{Case 2:}

For this case, the solution interval is chosen as $x=[-10,10]$ and $T=1$. Also, the parameters seen in the model are chosen as $\kappa=\mu=1, r=0.8$ [30]. Similar to case 1, Table 5 is prepared to check effect of time partition on numerical solution. Thus, Table is prepared for various values of $\Delta t$. In table 6, numerical solutions of FitzHugh-Nagumo model for several values of fractional derivative are demonstrated.

Figures show numerical behaviour of Time fractional FitzHugh-Nagumo model for $r=0.8, \gamma=0.9, \Delta t=$ $0.01, N=400$ and different final times in. Figure clears behaviour of model at final time $T=5$, and is the 3-dimensional representation of the model. 
Table 5. Case 2: Error norms $L_{2}$ and $L_{\infty}$ for different values of $\Delta t$ and $\gamma$ for $N=400$.

\begin{tabular}{lcccc}
\hline \hline$N=400$ & $\gamma=0.5$ & \multicolumn{2}{c}{$\gamma=0.9$} \\
\hline$\Delta t$ & $L_{2} \times 10^{3}$ & $L_{\infty} \times 10^{3}$ & $L_{2} \times 10^{3}$ & $L_{\infty} \times 10^{3}$ \\
\hline 0.1 & 11.9763931 & 7.29847571 & 5.10996928 & 3.09912528 \\
0.01 & 8.30005134 & 5.34968587 & 3.10841816 & 2.02770504 \\
0.005 & 8.10092924 & 5.24171639 & 2.96691736 & 1.94862517 \\
0.0025 & 8.00168118 & 5.18768771 & 2.89126522 & 1.90592140 \\
0.001 & 7.94222728 & 5.15525135 & 2.84271156 & 1.87833803 \\
\hline \hline
\end{tabular}

Table 6. Case 2: A comparison between values of solutions of Time fractional FitzHugh-Nagumo model and absolute errors for $\gamma=0.8$ and $x=0.01$

\begin{tabular}{lllllll}
\hline \hline \multicolumn{7}{c}{$[30]$} \\
\hline \multicolumn{1}{c}{$t$} & $u_{R P S M}$ & $u_{H A M}$ & $u_{F V I M}$ & \multicolumn{1}{c}{$u_{N I M}$} & \multicolumn{1}{c}{$u_{F E M T}$} & $|u(x, t)-w(x, t)|_{F E M}$ \\
\hline 0.01 & 0.499745 & 0.499765 & 0.499774 & 0.497779 & 0.500038 & 0.0002929366 \\
0.05 & 0.494437 & 0.494699 & 0.494541 & 0.487317 & 0.495499 & 0.0010628858 \\
0.1 & 0.489004 & 0.489798 & 0.489186 & 0.476613 & 0.490853 & 0.0018553202 \\
0.15 & 0.484113 & 0.485631 & 0.484366 & 0.46698 & 0.486670 & 0.0025749224 \\
0.2 & 0.479543 & 0.481948 & 0.479864 & 0.457985 & 0.482762 & 0.0032545373 \\
\hline \hline
\end{tabular}

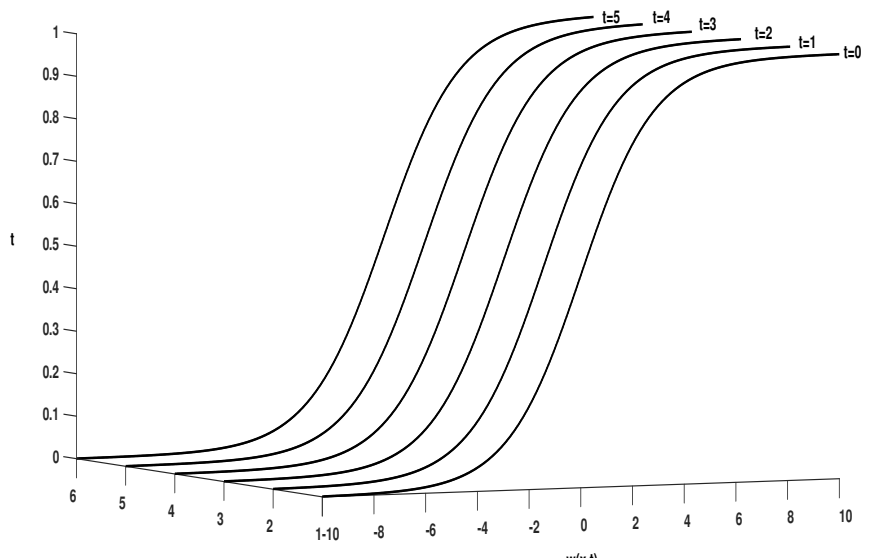

Figure 1. Numerical simulation of FitzHugh-Nagumo model: $r=0.8, \gamma=0.9, \Delta t=0.01, N=400$

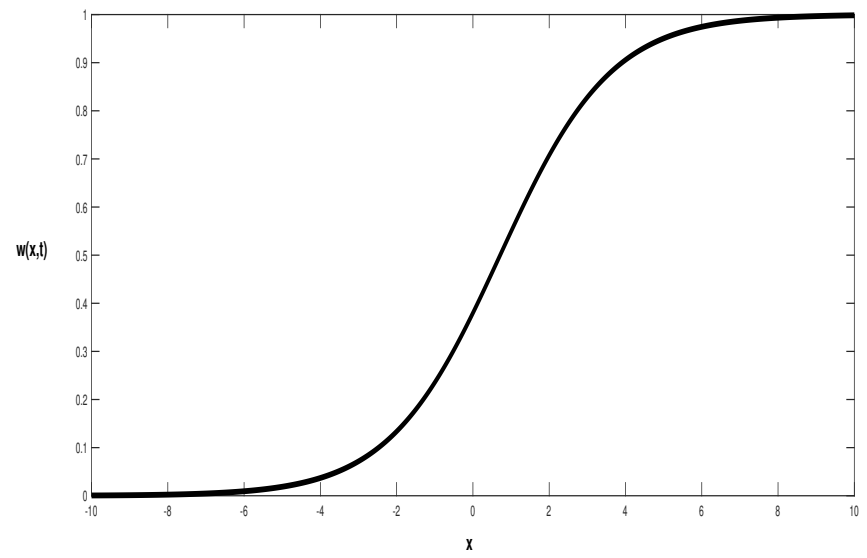

Figure 2. Numerical simulation of FitzHugh-Nagumo model: $r=0.8, \gamma=0.25, \Delta t=0.01, N=400$ 


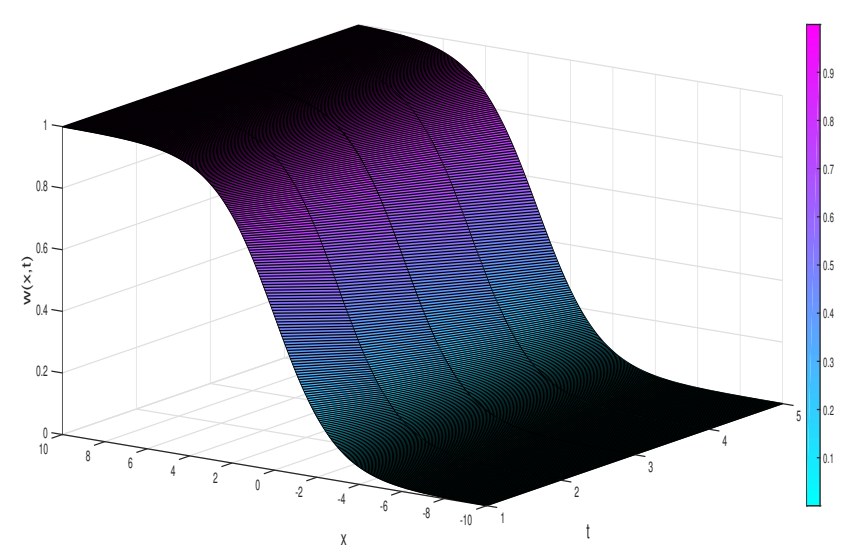

Figure 3. Numerical simulation of FitzHugh-Nagumo model: $r=0.8, \gamma=0.9, \Delta t=0.01, N=400$

\section{Conclusion}

In the present paper, finite element method based on trigonometric cubic B-spline is applied to time fractional FitzHugh-Nagumo model. Because the fractional derivative is taken in terms of Caputo sense, time discretization is made by $L 1$ algorithm. Numerical solutions with comparison tables and error norms are presented. As it is seen from the numerical results, the results demonstrate that finite element method with trigonometric cubic basis is accurate and effective among existing methods.

\section{Acknowledgment.}

We thank the reviewer for their insightful comments and suggestions that helped us improve the paper.

\section{Funding}

There is no funding for this work.

\section{Availability of data and materials}

Not applicable.

\section{Competing interests}

The authors declare that they have no competing interests.

\section{Author's contributions}

All authors contributed equally to the writing of this paper. All authors read and approved the final manuscript.

\section{References}

[1] Freed, A. D., Diethelm K.: Fractional calculus in biomechanics: a 3D viscoelastic model using regularized fractional derivative kernels with application to the human calcaneal fat pad. Biomechanics and modeling in mechanobiology. 5(4), 203-215 (2006).

[2] Ullah, N., Khan W., Wang S.: High performance direct torque control of electrical aerodynamics load simulator using fractional calculus. Acta Polytechnica Hungarica 11 (10), $59-78$ (2014).

[3] Baleanu, D., Atangana, A., Oukouomi Noutchie,S. C., Kurulay, M., Bildik, N.,Kilicman, A.: Fractional calculus: models and numerical methods. World Scientific, 3, 2012.

[4] Changpin, L., Zeng, F.: Numerical methods for fractional calculus. Chapman and Hall/CRC, 2019. 
[5] Oruç, Ö., Esen, A., Bulut F. : Highly accurate numerical scheme based on polynomial scaling functions for equal width equation. Wave Motion: 102760,(2021).

[6] Günerhan, H., Çelik, E.: Analytical and approximate solutions of fractional partial differential-algebraic equations. Applied Mathematics and Nonlinear Sciences 5 (1): 109-120 (2020).

[7] Mahdy, Amr M. S. :Numerical solutions for solving model time-fractional Fokker-Planck equation. Numerical Methods for Partial Differential Equations 37 (2), 1120-1135 (2021).

[8] Dokuyucu, M. A., Baleanu,D., Çelik E. : Analysis of Keller-Segel model with Atangana-Baleanu fractional derivative, Filomat 32 (16): 5633-5643 (2018).

[9] Owolabi, K. M., Hammouch, Z.: Spatiotemporal patterns in the Belousov-Zhabotinskii reaction systems with AtanganaBaleanu fractional order derivative. Physica A: Statistical Mechanics and its Applications 523, 1072-1090(2019).

[10] Onal, M., Esen, A. : A Crank-Nicolson approximation for the time fractional Burgers equation, Applied Mathematics and Nonlinear Sciences 5 (2), 177-184(2020).

[11] Oruç,Ö., Esen, A., Bulut, F.: A unified finite difference Chebyshev wavelet method for numerically solving time fractional Burgers' equation. Discrete \& Continuous Dynamical Systems-S 12 (3), 533(2019).

[12] Owolabi, Kolade M., Atangana A. : Analysis and application of new fractional Adams-Bashforth scheme with Caputo-Fabrizio derivative. Chaos, Solitons \& Fractals 105,111-119(2017).

[13] Oruç,Ö., Esen, A., Bulut, F.: A Haar wavelet approximation for two-dimensional time fractional reaction-subdiffusion equation, Engineering with Computers 35 (1), 75-86(2019).

[14] Owolabi, K. M.: Robust and adaptive techniques for numerical simulation of nonlinear partial differential equations of fractional order. Communications in Nonlinear Science and Numerical Simulation 44, 304-317(2017).

[15] FitzHugh R.: Mathematical models of threshold phenomena in the nerve membrane. Bull. Math. Biophysics, 17,257278(1955)

[16] Nagumo J., Arimoto S., Yoshizawa S: An active pulse transmission line simulating nerve axon. Proc. IRE. 50,20612070(1962).

[17] Xu, B., Binczak, S., Jacquir, S., Pont, O., Yahia, H.: Parameters analysis of FitzHugh-Nagumo model for a reliable simulation. Annu Int Conf IEEE Eng Med Biol Soc. 2014;2014:4334-7. doi: 10.1109/EMBC.2014.6944583. PMID: 25570951.

[18] Alphen, F. I. M: Model order reduction on FitzHugh-Nagumo model. BS thesis. University of Twente, 2020.

[19] Singh, S. : Mixed-Type Discontinuous Galerkin Approach for Solving the Generalized FitzHugh-Nagumo ReactionDiffusion Model. International Journal of Applied and Computational Mathematics 7 (5), 1-16 (2021).

[20] Yongheng, W., Cai, L., Feng, X., Luo,X., Gao, H.: A ghost structure finite difference method for a fractional FitzHughNagumo monodomain model on moving irregular domain. Journal of Computational Physics 428, 110081 (2021).

[21] Hemami, M., Parand,K., Rad J. A.: An efficient meshfree machine learning approach to simulate the generalized Fitzhugh-Nagumo equation inspired by neuroscience. In: The 51th Annual Iranian Mathematics Conference, 16-19 February, University of Kashan .1-4,(2021)

[22] Jiwari, R., Gupta, R. K., Kumar V.: Polynomial differential quadrature method for numerical solutions of the generalized Fitzhugh-Nagumo equation with time-dependent coefficients. Ain Shams Engineering Journal 5 (4), 1343-1350(2014).

[23] Liu, F., Turner, I., Yang, Q., Burrage, K.:A numerical method for the fractional Fitzhugh-Nagumo monodomain model. Anziam Journal 54, C608-C629(2012).

[24] Kumar, D., Singh,J., Baleanu, D.:A new numerical algorithm for fractional Fitzhugh-Nagumo equation arising in transmission of nerve impulses. Nonlinear Dynamics 91 (1), 307-317(2018).

[25] Injrou, S., Mahmoud, S., Kassem, A.:A Stable Numerical Scheme for Fitzhugh-Nagumo Time-Fractional Partial Differential Equation. Tishreen University Journal-Basic Sciences Series 41 (4), (2019). 
[26] Chapwanya, M., Jejeniwa, O. A., Appadu, A. R., Lubuma, J. M.-S.: An explicit nonstandard finite difference scheme for the FitzHugh-Nagumo equations. International Journal of Computer Mathematics 96 (10), 1993-2009(2019).

[27] Jiwari, R., Gupta, R. K., Kumar, V.:Polynomial differential quadrature method for numerical solutions of the generalized Fitzhugh-Nagumo equation with time-dependent coefficients. Ain Shams Engineering Journal 5 (4), 1343-1350(2014).

[28] Ersoy Hepson, O., Dağ, İ, Saka B., Ay B.:The Cubic B-spline Least Squares Finite Element Method for the Numerical Solutions of Regularized Long Wave Equation. International Journal of Computer Mathematics, 1-12 (2021). https://doi.org/10.1080/00207160.2021.1940979

[29] Yuste, Santos B., Acedo, L. An explicit finite difference method and a new von Neumann-type stability analysis for fractional diffusion equations. SIAM Journal on Numerical Analysis 42 (5), 1862-1874(2005).

[30] Tchier, F., Inc M., Korpinar Z.S.:Solutions of the time fractional reaction-diffusion equations with residual power series method. Advances in Mechanical Engineering 8 (10) : 1687814016670867(2016).

\section{Affiliations}

BERAT KARAAGAC

AdDRESS: Adıyaman University, Dept. of Mathematics Education, Adıyaman-Turkey.

E-MAIL: bkaraagac@adiyaman.edu.tr

ORCID ID:0000-0002-6020-3243 